\title{
Application of Raspberry Pi and PIR Sensor for Monitoring of Smart Surveillance System
}

\author{
Yogita Vijay Narkhede ${ }^{1}$, S. G. Khadke ${ }^{2}$ \\ ${ }^{1}$ G. H. Raisoni College of Engineering, Jalgaon \\ ${ }^{2}$ G. H. Raisoni College of Engineering, Department Of Electronics, Jalgaon
}

\begin{abstract}
This paper represents the design and implementation of smart surveillance monitoring system using Raspberry pi and PIR sensor for mobile devices. It increases the usage of mobile technology to provide essential security to homes and other control applications. The proposed house security system captures information and transmits it through a $3 G$ modem to a smart phone using web application. Raspberry pi operates and controls motion detectors; video camera for remote sensing, surveillance, streams live video and records it for future playback. Infrared sensor detects the located persons. For example, when motion is detected, the cameras starts recording and the Raspberry pi device alerts the possible illicit having a smart phone. Raspberry-pi includes two main components: 1Web Application that executes on the mobile device's browser.2- the server-side scripts that operated by the Raspberry Pi Hardware tool component run in a cloud.
\end{abstract}

Keyword: USB Cameras, PIR sensor, Raspberry Pi, Relay, 3G dongle, Smart phone

\section{Introduction}

Raspberry pi is similar to credit-card sized computer. It functions as a computer. There are various surveillance systems like camera, CCTV etc. In such types of surveillance systems, the person who is stationary and is located in that specific area can only see what is happening in that place. Whereas, here, even if the user is moving from one place to another, he/she can keep way of what is happening in that particular place. Another advantage is that it provides privacy on both sides which is viewed by only one person. The other advantage is a simple circuit. The Raspbian OS used as a operating system. Raspbian OS has to be installed so that the image can be transmitted to the smartphone.

Temperature, Humidity and Human motions are one of the most frequently observed parameters, and are extremely harmful to temperature, humidity and PIR values for a long times. So proposed system capable to monitoring these values indefinitely without any delay and putting any harm's way. Our proposed system is implemented on Raspberry pi and interfaced with four sensors and controlling the device also live video streaming is implemented for quick actions.

\section{Methodology}

Surveillance means monitoring the people's changing information like activities behaviour for the purpose of protecting, managing and influencing. The surveillance is a French word which means "watching over". Actually the surveillance means watching over from a distance by using electronic equipment such as CCTV cameras. The functions of various components are given below:

\subsection{USB Camera}

USB Camera captures the image and sends t to the USB port of the Raspberry Pi board. The camera model used here is USB camera model 2.0.

\subsection{Raspberry pi}

Raspberry pi is a small credit-card sized computer capable of performing various functions such as in surveillance systems, military applications, etc. The various components of Raspberry-Pi are

\subsubsection{SD Card Slot}

It is used to install OS/booting/long term storage. The total memory of the SD card is about $8 \mathrm{~GB}$.

\subsubsection{Micro USB Power Port provides 700mA at 5A.}

\subsubsection{RCA Video Out}

It is connected to display if HDMI output is not used. It is used to carry audio and video signals. They are otherwise called as $\mathrm{A} / \mathrm{V}$ jacks.

\subsubsection{Audio Out}

Digital audio is obtained if HDMI is used by obtaining stereo audio. Here analogue RCA connection is used.

\subsubsection{Ethernet Port}

It is used to connect to the Internet. It also plays a role in updating, getting new software easier.

\subsubsection{HDMI OUT (High Definition Multimedia Interface)} It is used with HDTV"s and monitors with HDMI input. Also HDMI-HDMI is used here.

\subsubsection{BROADCOM BCM 2835}

It is otherwise defined as System on chip. It is a $700 \mathrm{MHz}$ Processor. It has a video core IV GPU.

\subsubsection{GPIO}

It allows us to control and interact with real world. 


\section{International Journal of Science and Research (IJSR) \\ ISSN (Online): 2319-7064}

Index Copernicus Value (2013): 6.14 | Impact Factor (2014): 5.611

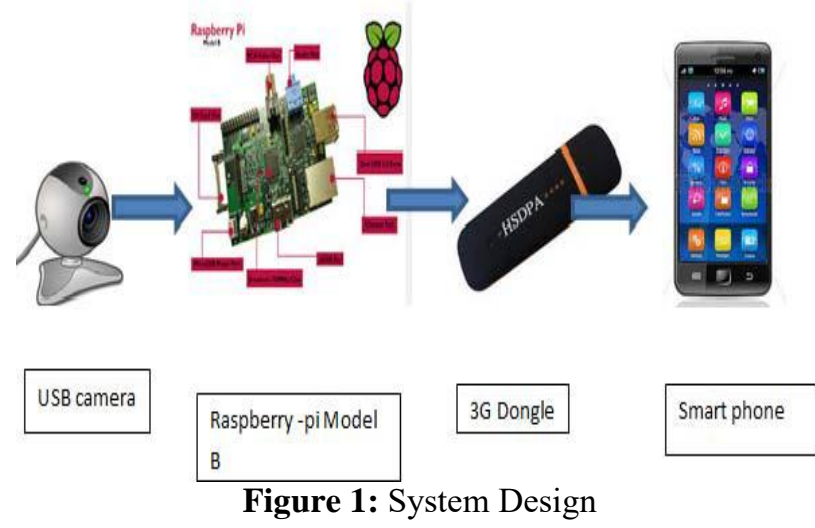

Figure 1 shows, Raspberry PI is connected to the USB camera with the help of USB port. Raspbian operating System is installed in the PC. Raspberry-pi works only on Linux operating system. Raspbian is a free operating system based on Debian optimization of the Raspberry Pi hardware. An operating system is the set of basic programs and utilities that make Raspberry Pi run. However, Raspbian provides a pure OS: it comes with over 35,000 packages, pre-compiled software bundled together to make easy installation on your Raspberry Pi.

\section{Conclusion}

Thus we designed a smart surveillance system capable of recording/ capturing video/image and transmitting to a smart phone. It provides advantage by offering reliability and privacy on both sides. It is authenticated and encrypted on the receiver side; hence it supports only the person concerned to view the details. Necessary action can be taken in short time in the case of emergency conditions such as elderly person falling sick, military areas, smart homes, offices, industries etc.

\section{References}

[1] Sharma, Rupam Kumar, et al."Android interface based GSM home security system." Issues and Challenges in intelligent Computing Techniques (ICICT), 2014 International Conference on. IEEE, 2014.

[2] De Luca, Gabriele, et al. "The use of NFC and Android technologies to enable a KNX-based smart home." Software, Telecommunications and Computer Networks (SoftCOM), 2013 21st International Conference on. IEEE, 2013.

[3] Gu, Yi, et al. "Design and implementation of UPnPBased Surveillance Camera System for Home Security."Information Science and Applications (ICISA), 2013 International Conference on IEEE, 2013.

[4] Van Thanh Trung, Bui, and Nguyen Van Cuong."Monitoring and controlling devices system by GPRS on FPGA platform." Advanced Technologies for Communications (ATC), 2013 International Conference on. IEEE, 2013.

[5] Karia, Deepak, et al."Performance analysis of Zigbee based Load Contorl and power monitoring system." Advances in Computing, Communications and Informatics (ICACCI), 2013 International Conference on. IEEE, 2013.
[6] Tupakula, Udaya, Vijay Varadharajan, and Sunil Kumar Vuppala."Security Techniques for Beyond 3G Wireless Mobile Networks." Embedded Ubiquitous Computing (EUC), 2011 IFIP 9th International Conference on. IEEE, 2011 . 